PROCEEDINGS OF THE

AMERICAN MATHEMATICAL SOCIETY

Volume 140, Number 3, March 2012, Pages 839-845

S 0002-9939(2011)11435-7

Article electronically published on November 2, 2011

\title{
PRIME END ROTATION NUMBERS OF INVARIANT SEPARATING CONTINUA OF ANNULAR HOMEOMORPHISMS
}

\author{
SHIGENORI MATSUMOTO \\ (Communicated by Yingfei Yi)
}

\begin{abstract}
Let $f$ be a homeomorphism of the closed annulus $A$ isotopic to the identity, and let $X \subset \operatorname{Int} A$ be an $f$-invariant continuum which separates $A$ into two domains, the upper domain $U_{+}$and the lower domain $U_{-}$. Fixing a lift of $f$ to the universal cover of $A$, one defines the rotation set $\tilde{\rho}(X)$ of $X$ by means of the invariant probabilities on $X$, as well as the prime end rotation number $\check{\rho}_{ \pm}$of $U_{ \pm}$. The purpose of this paper is to show that $\check{\rho}_{ \pm}$belongs to $\tilde{\rho}(X)$ for any separating invariant continuum $X$.
\end{abstract}

\section{INTRODUCTION}

Let $f$ be a homeomorphism of the closed annulus $A=S^{1} \times[-1,1]$, isotopic to the identity; i.e. $f$ preserves the orientation and each of the boundary components $\partial_{ \pm} A=S^{1} \times\{ \pm 1\}$. Suppose there is an $f$-invariant partition of $A ; A=U_{-} \cup X \cup U_{+}$, where $U_{ \pm}$is a connected open set containing the boundary component $\partial_{ \pm} A$ and $X$ is a connected compact set. Let

$$
\pi: \tilde{A}=\mathbb{R} \times[-1,1] \rightarrow S^{1} \times[-1,1]
$$

be the universal covering map and $T: \tilde{A} \rightarrow \tilde{A}$ a generator of the covering transformation group, $T(\xi, \eta)=(\xi+1, \eta)$. Denote by $p: \tilde{A} \rightarrow \mathbb{R}$ the projection onto the first factor.

Fix once and for all a lift $\tilde{f}: \tilde{A} \rightarrow \tilde{A}$ of $f$. Then the function $p \circ \tilde{f}-p$ is $T$-invariant and can be looked upon as a function on the annulus $A$. Define the rotation set $\tilde{\rho}(X)$ as the set of values $\mu(p \circ \tilde{f}-p)$, where $\mu$ ranges over the $f$-invariant probability measures supported on $X$. The rotation set is a compact interval (maybe one point) in $\mathbb{R}$, which depends upon the choice of the lift $\tilde{f}$ of $f$.

The first example of an invariant continuum $X$ such that the frontiers of $U_{ \pm}$ satisfy $\operatorname{Fr}\left(U_{+}\right)=\operatorname{Fr}\left(U_{-}\right)=X$ and that the rotation set $\tilde{\rho}(X)$ is not a singleton was constructed by G. D. Birkhoff in his 1932 paper [B] and is referred to as a Birkhoff attractor. It turns out that the Birkhoff attractor is an indecomposable continuum ([C, L2]). Furthermore it is shown by P. Le Calvez ([L1]) that any rational number between the two prime end rotation numbers is realized by a corresponding periodic point of $\tilde{f}$.

Received by the editors December 5, 2010.

2010 Mathematics Subject Classification. Primary 37E30; Secondary 37E45.

Key words and phrases. Continuum, rotation set, prime end rotation number, Brouwer line, foliations.

The author was partially supported by Grant-in-Aid for Scientific Research (C) No. 20540096.

(C)2011 American Mathematical Society 839

Reverts to public domain 28 years from publication 
Let $\hat{U}_{ \pm}=U_{ \pm} \cup \partial_{\infty} U_{ \pm}$be the prime end compactification of $U_{ \pm}$, where $\partial_{\infty} U_{ \pm}$is the space of the prime ends ([E, $, \bar{M}, \overline{M N}])$. The space $\partial_{\infty} U_{ \pm}$is homeomorphic to the circle and $\hat{U}_{ \pm}$to the closed annulus. As is well known, the homeomorphism $f$ restricted to $U_{ \pm}$extends to a homeomorphism $\hat{f}_{ \pm}: \hat{U}_{ \pm} \rightarrow \hat{U}_{ \pm}$. Denoting $I_{+}=[0,1]$ and $I_{-}=[-1,0]$, define a homeomorphism

$$
\Psi_{ \pm}: \hat{U}_{ \pm} \rightarrow S^{1} \times I_{ \pm}
$$

such that $\Psi_{ \pm}\left(\partial_{\infty} U_{ \pm}\right)=S^{1} \times 0$. By some abuse of notation denote by $\pi: \check{U}_{ \pm} \rightarrow \hat{U}_{ \pm}$ the universal covering map. Thus $\pi^{-1}\left(U_{ \pm}\right)$is considered to be a subspace of both $\tilde{A}$ and $\check{U}_{ \pm}$. Let $\check{f}_{ \pm}: \check{U}_{ \pm} \rightarrow \check{U}_{ \pm}$be the lift of $\hat{f}_{ \pm}$such that $\check{f}_{ \pm}=\tilde{f}$ on $\pi^{-1}\left(U_{ \pm}\right)$. The rotation number of the restriction of $\check{f}_{ \pm}$to $\pi^{-1}\left(\partial_{\infty} U_{ \pm}\right)$, denoted by $\check{\rho}_{ \pm}$, is called the prime end rotation number of $U_{ \pm}$.

The purpose of this paper is to show the following.

Theorem 1. The prime end rotation number $\check{\rho}_{ \pm}$belongs to $\tilde{\rho}(X)$.

This result is already known for $X=\operatorname{Fr}\left(U_{-}\right)=\operatorname{Fr}\left(U_{+}\right)([\mathrm{BG}])$, and for any $X$ if the homeomorphism $f$ is area preserving (Lemma 5.4, [FL]).

It is shown in Theorem 2.2 of $[\mathrm{F}$. that any rational number in $\tilde{\rho}(X)$ is realized by a periodic point if $X$ consists of nonwandering points. Notice then that $X$, consisting of chain recurrent points, is chain transitive since it is connected, and thus satisfies the condition of Theorem 2.2. As a corollary we have

Corollary 2. If $X$ consists of nonwandering points and if $p / q$ lies in the closed interval bounded by $\check{\rho}_{-}$and $\check{\rho}_{+}$, then there is a point $x \in \pi^{-1}(X)$ such that $\tilde{f}^{q}(x)=$ $T^{p}(x)$.

In what follows we also use the following notation. Let

$$
\check{\Psi}_{ \pm}: \check{U}_{ \pm} \rightarrow \mathbb{R} \times I_{ \pm}
$$

be a lift of $\Psi_{ \pm}$, and define $\check{p}_{ \pm}: \check{U}_{ \pm} \rightarrow \mathbb{R}$ by $\check{p}_{ \pm}=p \circ \check{\Psi}_{ \pm}$. The projection $\check{p}_{ \pm}$is within a bounded error of $p$ on $\pi^{-1}(C)$ for a compact domain $C$ of $U_{ \pm}$. However, they may be quite different on the whole $\pi^{-1}\left(U_{ \pm}\right)$.

\section{ProOF}

First of all let us state a deep and quite useful theorem of P. Le Calvez ([L3]) which plays a key role in the proof. A fixed point free and orientation preserving homeomorphism $F$ of the plane $\mathbb{R}^{2}$ is called a Brouwer homeomorphism. A proper oriented simple curve $\gamma: \mathbb{R} \rightarrow \mathbb{R}^{2}$ is called a Brouwer line for $F$ if $F(\gamma) \subset R(\gamma)$ and $F^{-1}(\gamma) \subset L(\gamma)$, where $R(\gamma)$ (resp. $L(\gamma)$ ) is the right (resp. left) side complementary domain of $\gamma$, which is decided by the orientation of $\gamma$.

Theorem 2.1. Let $F$ be a Brouwer homeomorphism commuting with the elements of a group $\Gamma$ which acts on $\mathbb{R}^{2}$ freely and properly discontinuously. Then there is $a \Gamma$-invariant oriented topological foliation of $\mathbb{R}^{2}$ whose leaves are Brouwer lines of $F$.

The proof of Theorem 1 is by absurdity. Assume by way of contradiction that $\check{\rho}_{-}<p / q<\inf \tilde{\rho}(X)$. Considering $\tilde{f}^{q} T^{-p}$ instead of $\tilde{f}$, it suffices to deduce a contradiction under the following assumption.

Assumption 2.2. $\check{\rho}_{-}<0<\inf \tilde{\rho}(X)$. 
Since $\inf \tilde{\rho}(X)>0$, the map $\tilde{f}$ does not admit a fixed point in $\pi^{-1}(X)$. The overall strategy of the proof is to modify the homeomorphism $f$ away from $X$ to a new one $g$ without creating fixed points in $A$ such that the restrictions of $\tilde{g}$ to the lifts of both boundary circles $\pi^{-1}\left(\partial_{ \pm} A\right)$ are nontrivial rigid translations by the same translation number. Then by glueing the two boundary circles we obtain a torus $T^{2}$ and a homeomorphism on $T^{2}$. Now we can apply Theorem 2.1 to the lift of the homeomorphism to the universal covering space. This yields a topological foliation on $T^{2}$, which has long been well understood. The proof will be done by analyzing the foliation. We first prepare a lemma which is necessary for the desired modification. We do not presume Assumption 2.2 in the following.

Lemma 2.3. Assume $\tilde{f}$ does not admit a fixed point in $\pi^{-1}(X)$. Then the prime end rotation number $\check{\rho}_{ \pm}$is nonzero.

Proof. Consider the mapping $\tilde{f}$ - Id defined on $\tilde{A}$. Since it is $T$-invariant, it yields a mapping from $A$, still denoted by the same letter. Then since there is no fixed point of $\tilde{f}$ in $X$, we have $(\tilde{f}-\mathrm{Id})(X) \subset \mathbb{R}^{2} \backslash\{0\}$. Therefore there is an annular open neighbourhood $V$ of $X$ for which we get a mapping

$$
\tilde{f}-\mathrm{Id}: V \rightarrow \mathbb{R}^{2} \backslash\{0\} .
$$

Clearly for any positively oriented essential simple closed curve $\gamma$ in $V$, the degree of the map

$$
\tilde{f}-\mathrm{Id}: \gamma \rightarrow \mathbb{R}^{2} \backslash\{0\}
$$

must be the same. If the curve $\gamma$ is contained in $U_{ \pm}$, then the degree can be studied by considering the map $\check{f}_{ \pm}$defined on the lift $\check{U}_{ \pm}$of the prime end compactification $\hat{U}_{ \pm}$. If the prime end rotation number $\check{\rho}_{ \pm}$is nonzero, the degree is clearly 0 . Notice that our definition of the degree differs from the usual definition of the index.

To analyze the case $\check{\rho}_{ \pm}=0$, we need the following form of the CartwrightLittlewood theorem CL.

Theorem 2.4. If $\check{\rho}_{+}=0$ and if $\operatorname{Fix}(\tilde{f}) \cap \pi^{-1}(X)=\emptyset$, then the map $\hat{f}_{+}$on $\partial_{\infty} U_{+}$ is Morse Smale and the attractors (resp. repellors) of $\left.\hat{f}_{+}\right|_{\partial_{\infty} U_{+}}$are attractors (resp. repellors) of the whole map $\hat{f}_{+}$.

This is slightly stronger than the usual version in which it is assumed that $\operatorname{Fix}(f) \cap X=\emptyset$. However, the proof works as well under the assumption of Theorem 2.4 See e.g. Section 3 of $\mathrm{MN}$.

Let us complete the proof of Lemma 2.3. Theorem 2.4 enables us to compute the degree of the curve $\delta$ in $U_{ \pm}$when $\check{\rho}_{ \pm}=0$. The degree is $n$ if $\delta \subset U_{-}$and $-n$ if $\delta \subset U_{+}$, where $n$ is the number of the attractors. Since the degree must be the same in $U_{-}$and $U_{+}$, the conclusion follows.

Now we have $\check{\rho}_{-}<0$ and $\check{\rho}_{+} \neq 0$ by Assumption 2.2 and Lemma 2.3. Let us start the modification of $f$.

Lemma 2.5. Under Assumption 2.2, there exists a homeomorphism $g$ of A such that:

(1) $g=f$ in some neighbourhood of $X$,

(2) $\tilde{g}$ does not admit a fixed point in $\tilde{A}$, where $\tilde{g}$ is the lift of $g$ such that $\tilde{g}=\tilde{f}$ on $\pi^{-1}(X)$, 
(3) $\tilde{g}$ is a negative rigid translation by the same translation number on $\pi^{-1}\left(\partial_{ \pm} A\right)$, and

(4) $\check{p}_{-} \circ \check{g}_{-}-\check{p}_{-} \leq-c$ on $\hat{U}_{-}$for some positive number $c$.

Proof. The modification in $U_{-}$will be done in the following way. We identify $\hat{U}_{-}$ with $S^{1} \times[-1,0]$ by the homeomorphism $\Psi_{-}$and the universal covering space $\check{U}_{-}$ with $\mathbb{R} \times[-1,0]$. Thus $\check{p}_{-}$is just the projection onto the first factor, $\check{p}_{-}(\xi, \eta)=\xi$. Since $\check{\rho}_{-}<0$, the lift

$$
\check{f}_{-}: \mathbb{R} \times[-1,0] \rightarrow \mathbb{R} \times[-1,0]
$$

of $\hat{f}_{-}$satisfies that $\check{p}_{-} \circ \check{f}_{-}(\xi, 0)<\xi-2 c$ for some $c>0$. Therefore changing the coordinates of $[-1,0]$ if necessary, one may assume that $\check{p}_{-} \circ \check{f}_{-}(\xi, \eta) \leq \xi-c$ if $(\xi, \eta) \in \mathbb{R} \times[-1 / 2,0]$. Define a homeomorphism $h$ of $S^{1} \times[-1,0]$ by

$$
h(\xi, \eta)=(\xi+\varphi(\eta) \bmod 1, \eta),
$$

where $\varphi:[-1,0] \rightarrow(-\infty, 0]$ is a continuous function such that $\varphi([-1 / 2,0])=0$ and

$$
\varphi(\eta) \leq-\sup \left\{\left(\check{p}_{-} \circ \check{f}_{-}-\check{p}_{-}\right)(\xi, \eta) \mid \xi \in S^{1}\right\}-c .
$$

Define $g=f \circ h$. Then its lift $\check{g}_{-}$satisfies

$$
\check{p}_{-} \circ \check{g}_{-}-\check{p}_{-} \leq-c
$$

on $\check{U}_{-}=\mathbb{R} \times[-1,0]$. Clearly condition $(3)$ for $\pi^{-1}\left(\partial_{-} A\right)$ can be established by a further obvious modification.

Now to modify $f$ in $U_{+}$, we do the same thing as in $U_{-}$. If the prime end rotation number $\check{\rho}_{+}$is negative, then with an auxiliary modification we are done. If it is positive, insert a time one map of the Reeb flow.

Consider the torus $T^{2}$ which is obtained from $A$ by glueing the two boundary curves $\partial_{-} A$ and $\partial_{+} A$. Then condition (3) above shows that $g$ induces a homeomorphism of $T^{2}$, again denoted by $g$. The universal cover of $T^{2}$ is $\mathbb{R}^{2}$, and $\tilde{A}=\mathbb{R} \times[-1,1]$ is a subset of $\mathbb{R}^{2}$. The lift $\tilde{g}: \tilde{A} \rightarrow \tilde{A}$ can be extended uniquely to a lift $\tilde{g}: \mathbb{R}^{2} \rightarrow \mathbb{R}^{2}$ of $g: T^{2} \rightarrow T^{2}$. The covering transformation group $\Gamma$ is isomorphic to $\mathbb{Z}^{2}$, generated by the horizontal translation $T$ and the vertical translation by 2 , denoted by $S$. Since $\tilde{g}$ is a Brouwer homeomorphism which commutes with $\Gamma$, there is a $\Gamma$-invariant oriented foliation on $\mathbb{R}^{2}$ whose leaves are Brouwer lines for $\tilde{g}$. This yields an oriented foliation $\mathcal{F}$ on the torus $T^{2}$. The proof is divided into several cases according to the topological type of the foliation $\mathcal{F}$. We are going to deduce a contradiction in each case. However, before going into detail we need another lemma.

Lemma 2.6. For any $C>0$ there is $n>0$ such that $p \circ \tilde{g}^{n}-p \geq C$ on $X$.

Proof. If not, there would be a point $x_{n} \in X$ for any $n>0$ such that

$$
\left(p \circ \tilde{g}^{n}-p\right)\left(x_{n}\right)=\sum_{j=0}^{n-1}(p \circ \tilde{g}-p)\left(g^{j}\left(x_{n}\right)\right)<C
$$

for some $C>0$, and the averages of Dirac masses

$$
\mu_{n}=\frac{1}{n} \sum_{j=0}^{n-1} g_{*}^{j} \delta_{x_{n}}
$$


would satisfy $\mu_{n}(p \circ \tilde{g}-p)<C / n$. Therefore an accumulation point $\mu$ of $\mu_{n}$ would have the property that $\mu(p \circ \tilde{g}-p) \leq 0$, contradicting the assumption $\inf \tilde{\rho}(X)>$ 0 .

Case 1. The foliation $\mathcal{F}$ does not admit a compact leaf. Then $\mathcal{F}$ is conjugate either to a linear foliation or to a Denjoy foliation, both of irrational slope. The lift $\tilde{\mathcal{F}}$ of $\mathcal{F}$ to the open annulus $\mathbb{R}^{2} /\langle T\rangle$ is conjugate to a foliation by vertical lines. The space of leaves of $\tilde{\mathcal{F}}$ is homeomorphic to $S^{1}$, and there is a projection from $\mathbb{R}^{2} /\langle T\rangle$ to $S^{1}$ along the leaves of the foliation. This lifts to a projection $q: \mathbb{R}^{2} \rightarrow \mathbb{R}$.

Now $q$ restricted to $\tilde{A}$ is within a bounded error of the first factor projection $p: \tilde{A} \rightarrow \mathbb{R}$ that we have used for the definition of the rotation set $\tilde{\rho}(X)$. In fact both $p$ and $q$ are lifts of degree one maps from $\mathbb{R}^{2} /\langle T\rangle$ to $S^{1}$, and their difference is bounded on the preimage $\tilde{A}=\pi^{-1}(A)$ of a compact subset $A$. Thus Lemma 2.6 shows that $q \circ \tilde{g}^{n}(x) \rightarrow \infty(n \rightarrow \infty)$ for $x \in \pi^{-1}(X)$. That is, the foliation $\tilde{\mathcal{F}}$ is oriented upward. But this shows that $q \circ \tilde{g}(x)>q(x)$ even for a point $x \in \pi^{-1}\left(\partial_{-} A\right)$. On the other hand, by condition (3) of Lemma 2.5, $\tilde{g}$ is a negative translation on $\pi^{-1}\left(\partial_{-} A\right)$, a contradiction.

Case 2.1. The foliation $\mathcal{F}$ admits a compact leaf $L$ of nonzero slope and does not admit a Reeb component. In this case the lifted foliation $\tilde{\mathcal{F}}$ is also conjugate to the vertical foliation, and the argument of Case 1 applies.

Case 2.2. The foliation $\mathcal{F}$ admits a Reeb component $R$ of nonzero slope. The Brouwer property of leaves implies that $g(R) \subset \operatorname{Int}(R)$ or $g^{-1}(R) \subset \operatorname{Int}(R)$. That is, a point of the boundary of $R$ is wandering under $g$. Therefore $\partial_{-} A$, consisting of nonwandering points of $g$ according to (3) of Lemma 2.5, cannot intersect the boundary of $R$, which is however impossible since the slope of $R$ is nonzero.

Case 2.3. The foliation $\mathcal{F}$ admits a compact leaf of slope 0 . Hereafter we only consider the dynamics and the foliation on the open annulus $\mathbb{R}^{2} /\langle T\rangle$. Recall that $A$ is a subset of $\mathbb{R}^{2} /\langle T\rangle$, and the homeomorphism $g$ on $A$ is extended to the whole $\mathbb{R}^{2} /\langle T\rangle$, again denoted by $g$, in such a way that $g$ commutes with the vertical translation $S$, while the foliation is denoted by $\tilde{\mathcal{F}}$ as before.

Now the foliation $\tilde{\mathcal{F}}$ yields a partition $\mathcal{P}$ of the open annulus $\mathbb{R}^{2} /\langle T\rangle$ into compact leaves, interiors of Reeb components and foliated $I$-bundles. The set $\mathcal{P}$ is totally ordered by the height. The minimal element which intersects $X$ cannot be a compact leaf by the Brouwer line property. Let $R$ be the closure of the minimal element. Thus $R$ is either a Reeb component or a foliated $I$-bundle such that $\operatorname{Int}(R) \cap X \neq \emptyset$ and $\partial_{-} R \cap X=\emptyset$, where $\partial_{-} R$ is the lower boundary curve of $R$.

Assume for a while that $\partial_{-} R$ is oriented from the right to the left. Thus the homeomorphism $g$ carries $\partial_{-} R$ into the upper complement of $\partial_{-} R$.

Case 2.3.1. $R$ is a Reeb component. First notice that $g(R) \subset \operatorname{Int} R$ and that the interior leaves of $R$ are oriented upwards by the assumption $\inf \tilde{\rho}(X)>0$ and the fact that $g(X \cap R) \subset X \cap R$. Choose a simple arc

$$
\alpha:[0,1] \rightarrow \pi^{-1}(R)
$$

such that $\alpha(0) \in \pi^{-1}\left(\partial_{-} R\right), \alpha(1)=\tilde{g}(\alpha(0))$, and $\alpha((0,1)) \subset \operatorname{Int}\left(\pi^{-1}(R)\right) \backslash$ $\tilde{g}\left(\pi^{-1}(R)\right)$. Since $g^{-1}(\pi(\alpha))$ is below Int $R, \tilde{g}^{-1}(\alpha)$, and hence $\alpha$, is contained in $\pi^{-1}\left(U_{-}\right)$. 
Concatenating nonnegative iterates of $\alpha$, we obtain a simple path $\gamma:[0, \infty) \rightarrow$ $\pi^{-1}\left(R \cap U_{-}\right)$such that $\tilde{g} \circ \gamma(t)=\gamma(t+1)$ for any $t \geq 0$. Let $q: \pi^{-1}(\operatorname{Int}(R)) \rightarrow \mathbb{R}$ be the lift of the projection along the leaves. Since $\gamma([1, \infty))$ is contained in the lift of a compact subset $\tilde{g}(R) \subset \operatorname{Int}(R)$ and the leaves in $\operatorname{Int}(R)$ are oriented upward, we have $q \circ \gamma(t) \rightarrow \infty$ as $t \rightarrow \infty$. We also have $p \circ \gamma(t) \rightarrow \infty$ because $q$ is within bounded error of $p$ on $\gamma([1, \infty))$.

On the other hand, by condition (4) of Lemma 2.5] we have $\check{p} \circ \gamma(t) \rightarrow-\infty$ as $t \rightarrow \infty$. In particular, the curve $\gamma$ is proper both in $\tilde{A}$ and in $\check{U}_{-}$pointing toward the opposite direction. By joining the point $\gamma(0)$ to an appropriate point in $\pi^{-1}\left(\partial_{-} A\right)$, we obtain a simple curve $\delta$ in $\pi^{-1}\left(U_{-}\right)$starting at a point on $\pi^{-1}\left(\partial_{-} A\right)$ which extends $\gamma$.

Notice that there is a point of $\pi^{-1}(X)$ on the left of a proper oriented curve $\delta$ in $\tilde{A}$ because the map $p$ is bounded from below on $\delta$ and a high iterate of $T^{-1}$ carries a point in $\pi^{-1}(X)$ beyond that bound. (There might be a point of $\pi^{-1}(X)$ on the right of $\delta$ however.)

Let $x$ be a point in $\pi^{-1}\left(\partial_{-} A\right)$ left to the initial point of $\delta$. Then there is a simple path $\beta:[0, \infty) \rightarrow \pi^{-1}\left(U_{-}\right)$such that $\beta(0)=x, \lim _{t \rightarrow \infty} \beta(t) \in \pi^{-1}(X)$, and $\beta$ is disjoint from $\delta$. The path $\beta$, extendable in $\pi^{-1}(A)$, is also extendable in $\check{U}_{-}$, the lift of the prime end compactification. (See e.g. Lemma 2.5 of $[\mathrm{MN}]$.) This implies that $\beta$ defines a simple path in $\check{U}_{-}$joining $x$ to a prime end in $\pi^{-1}\left(\partial_{\infty} U_{-}\right)$ without intersecting $\delta$, which is impossible since $\pi^{-1}\left(\partial_{\infty} U_{-}\right)$is contained on the right side of the proper path $\delta$ in $\check{U}_{-}$since $\check{p}_{-} \delta(t) \rightarrow-\infty$, while $x$ is on the left side, a contradiction.

Case 2.3.2. $R$ is a foliated I-bundle. Thus the upper boundary curve $\partial_{+} R$ of $R$ is also oriented from the right to the left, and its image by $g$ lies on the upper complement of $R$. The interior leaves of $R$ are oriented upward.

Recall that the boundary component $\partial_{-} A$ consisting of nonwandering points cannot intersect a compact leaf. Moreover, $\partial_{-} A$ lies in a Reeb component or a foliated $I$-bundle whose interior leaves are oriented downward since $p \tilde{g}^{n}(x) \rightarrow-\infty$ as $n \rightarrow \infty$ for $x \in \pi^{-1}\left(\partial_{-} A\right)$. Let $C$ be the annulus in $\mathbb{R}^{2} /\langle T\rangle$ bounded by $\partial_{-} A$ and $\partial_{+} R$, the upper boundary curve of $R$. Notice that $\operatorname{Int}(C)$ contains $\partial_{-} R$.

Case 2.3.2.1. The intersection $X \cap C$ has a component which separates $\partial_{-} A$ from $\partial_{+} A$. One can derive a contradiction by the same argument as in Case 2.3.1, since the like defined path $\gamma$ cannot evade $R$.

Case 2.3.2.2. There is a simple path in $U_{-}$joining a point in $\partial_{-} A$ with a point in $\partial_{+} R$. Notice first of all that $g^{-1}(C) \subset C$. Let $\mathcal{Y}$ be the family of the connected components of $\pi^{-1}(X \cap C)$. Then any element $Y \in \mathcal{Y}$ is compact and intersects $\pi^{-1}\left(\partial_{+} R\right)$ since otherwise $Y$ would be a connected component of $\pi^{-1}(X)$ itself.

Choose a simple curve $\gamma:[0,1] \rightarrow \pi^{-1}(C)$ such that

(1) $\gamma(0) \in \pi^{-1}\left(\partial_{-} A\right)$,

(2) $\gamma(1) \in \pi^{-1}(X \cap C)$, and

(3) $\gamma([0,1)) \subset \pi^{-1}\left(U_{-} \cap C\right)$.

Let $Y$ be an element of $\mathcal{Y}$ which contains $\gamma(1)$. Then there are two unbounded connected components of the complement $\pi^{-1}(C) \backslash(Y \cup \gamma)$, one $L(Y \cup \gamma)$ on the left and the other $R(Y \cup \gamma)$ on the right.

Notice that for any $n>0, \tilde{g}^{-n} \gamma$ is a path in $C$, and that $p \tilde{g}^{-n}(\gamma(1)) \rightarrow-\infty$ and $p \tilde{g}^{-n}(\gamma(0)) \rightarrow \infty$ as $n \rightarrow \infty$. That is, for any large $n, \tilde{g}^{-n}(\gamma(1)) \in L(Y \cup \gamma)$ 
and $\tilde{g}^{-n}(\gamma(0)) \in R(Y \cup \gamma)$, showing that $\tilde{g}^{-n}(\gamma)$ intersects $\gamma$. On the other hand, in $\check{U}_{-}, \gamma$ defines a curve from a point in $\pi^{-1}\left(\partial_{-} A\right)$ to a prime end in $\pi^{-1}\left(\partial_{\infty} U_{-}\right)$. However, by condition (4) of Lemma 2.5, $\gamma$ cannot intersect $\tilde{g}^{-n}(\gamma)$ for any large $n$, a contradiction.

Finally the case where $\partial_{-} R$ is oriented from the left to the right can be dealt with similarly by reversing the time. This completes the proof of Theorem 1

\section{REFERENCES}

[B] G. D. Birkhoff, Sur quelques courbes fermées remarquables, Bull. Soc. Math. France 60(1932) 1-26; also in Collected Mathematical Papers of G. D. Birkhoff, vol. II, New York: Dover, 1968, pp. 444-461. MR 1504983

[BG] M. Barge and R. M. Gillete, Rotation and periodicity in plane separating continua, Ergod. Th. Dyn. Sys. 11(1991) 619-631. MR:1145613 (92m:54070)

[C] M. Charpentier, Sur quelques propriétés des courbes de M. Birkhoff, Bull. Soc. Math. France 62(1934) 193-224. MR.1505024

[CL] M. L. Cartwright and J. E. Littlewood, Some fixed point theorems, Ann. Math. 54(1951) 1-37. MR0042690(13:148f)

[E] D. B. A. Epstein, Prime ends, Proc. London Math. Soc. 42(1981) 385-414. MR614728 (83c:30025)

[F] J. Franks, Recurrence and fixed points of surface homeomorphisms, Ergod. Th. Dyn. Sys. 8(1988) 99-107. MR967632 (90d:58124)

[FL] J. Franks and P. Le Calvez, Regions of instability for non-twist maps, Ergod. Th. Dyn. Sys. 23(2003), 111-141. MR1971199 (2003m:37053)

[L1] P. Le Calvez, Existence d'orbits quasi-periodiques dans les attracteurs de Birkhoff, Commun. Math. Phys. 106(1986) 383-394. MR859817 (88a:58124)

[L2] P. Le Calvez, Propriétés des attracteurs de Birkhoff, Ergod. Th. Dyn. Sys. 8(1987) 241-310. MR951271 (90a:58103)

[L3] P. Le Calvez, Une version feuilletée équivariante du théorème de translation de Brouwer, Publ. Math. I. H. E. S. 102(2005) 1-98. MR2217051 (2007m:37100)

$[\mathrm{M}]$ J. Mather, Topological proofs of some purely topological consequences of Carathéodory's theory of prime ends, in: Th. M. Rassias, G. M. Rassias, eds., Selected Studies, NorthHolland (1982) 225-255. MR662863 (84k:57004)

[MN] S. Matsumoto and H. Nakayama, Continua as minimal sets of homeomorphisms of $S^{2}$, arXiv:1005.0360.

Department of Mathematics, College of Science and Technology, Nihon University, 1-8-14 Kanda, Surugadai, Chiyoda-Ku, Tokyo, 101-8308 Japan

E-mail address: matsumo@math.cst.nihon-u.ac.jp 